\title{
An experimental characterization of human falling down
}

\author{
Libo Meng ${ }^{1,2}$, Marco Ceccarelli ${ }^{3,4}$, Zhangguo Yu ${ }^{1,2,4}$, Xuechao Chen ${ }^{1,2,4}$, and Qiang Huang ${ }^{1,2,4}$ \\ ${ }^{1}$ Intelligent Robotics Institute, School of Mechatronical Engineering, Beijing Institute of Technology, \\ 5 Nandajie, Zhongguancun, Haidian, Beijing 100081, China \\ ${ }^{2}$ Key Laboratory of Biomimetic Robots and Systems, Ministry of Education, State Key Laboratory of \\ Intelligent Control and Decision of Complex Systems, Beijing Institute of Technology, 5 Nandajie, \\ Zhongguancun, Haidian, Beijing 100081, China \\ ${ }^{3}$ LARM: Laboratory of Robotics and Mechatronics, DICeM, University of Cassino and South Latium, Via Di \\ Biasio 43, 03043 Cassino, Fr, Italy \\ ${ }^{4}$ Beijing Advanced Innovation Center for Intelligent Robots and Systems, Beijing Institute of Technology, \\ China \\ Correspondence to: Xuechao Chen (chenxuechao@bit.edu.cn)
}

Received: 12 June 2016 - Revised: 7 January 2017 - Accepted: 9 March 2017 - Published: 29 March 2017

\begin{abstract}
This paper presents results of an experimental investigation on the falling down of the human body in order to identify significant characteristics and parameters. A specific lab layout has been settled up with vision tracking system and suitable sensors to monitor information on trajectories, impact force and acceleration during the falling with elaboration procedures that make fairly easy to track and interpret the motion characteristics. We focus on the more often falling mode: forward and backward falling Tests are discussed with results from lab tests that give both behavior and values of the biomechanics of falling down of the human body. Possible protection strategies for falling based on the proposed research are talked about at the last.
\end{abstract}

Tracking the motion of human is a major source of inspiration for understanding the characteristics of human motion and designing motion for robots. Gupta et al. recognize the human activities using the image sequences with action labels (Gupta et al., 2014). Roos built a walking model to predict the different risk of falling down for elder people (Roos et al., 2013). In the field of robotic, human motion data also provides a real inspiration for robot design and control. For instance, Huang et al. proposed a similarity evaluation between the human motion and robot motion and developed complex motion for the humanoid robot based on the evaluation (Huang et al., 2010). Zielinska et al. discussed the problem of using the human gaits to generate legged locomotion for biped robots. They were inspired by the biological Center of Gravity (COG) to produce leg joint trajectories (Zielinska et al., 2009). Zhao et al. design human-like motion for robotic arms using resolving the kinematic motion of human arm (Zhao et al., 2014).
In particular, falling down has attracted special interest. Such studies of human motion falling down can be found at the following researches. Robinovitch et al. have studied the human down for decades. They examined the situation of falling backward of a human. And the results showed that squatting during falling down decrease the impact severity. They also investigated the influence of two falling directions: forwards and backward, then, they used the video camera to analyze the falls in daily life (Hsiao et al., 1997; Sandler and Robinovitch, 2001; Robinovitch et al., 2004; Tan et al., 2006; Yang et al., 2013). Chen et al. proposed a biped model to capture and predict the falling cause (Chen et al., 2015). Hitcho et al. and Lee et al. studied the falling of elder person for home healthcare and hospital setting (Hitcho et al., 2004 and Lee et al., 2012). Ma et al. proposed a method of falling protected for humanoid robot inspired by the research of human falling down (Ma et al., 2014). Tomii et al. proposed a falling detection system using wireless sensors for elder people (Tomii et al., 2012). 


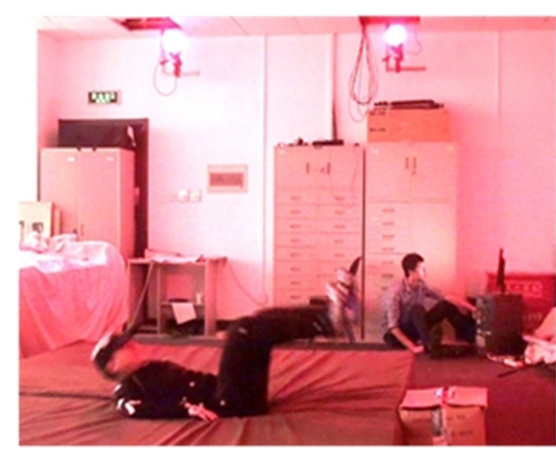

(a)

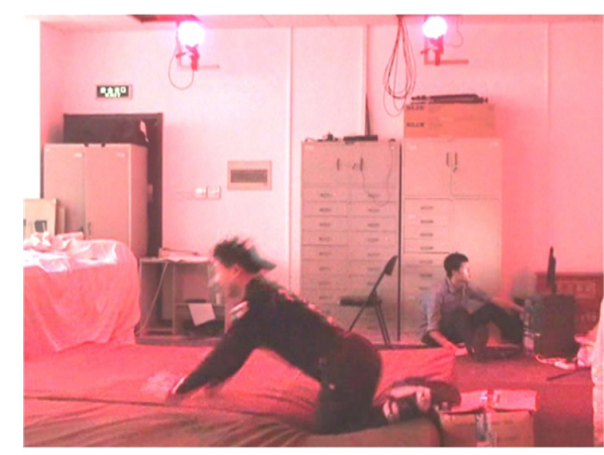

(b)

Figure 1. Human falling down in daily life: (a) falling backward, (b) falling forward.

Human motion can be acquired from different ways. Schmitz et al. used the motion capture system to measure the joints angle of human (Schmitz et al., 2014), Yang et al. introduced a method to analyze the 3-D human gait of through a markerless motion capture (Yang et al., 2014). Ayusawa et al. identified the inverse kinematics and geometric parameters using motion capture data (Ayusawa et al., 2014). Carnegie Mellon University (CMU) offered a valuable database of human motion, acquired by their vision tracking system, including walking, jumping, and sports and so on (Gross and Shi, 2001). Recently, some new studies recognize human motion including the human-object interactions and highly articulated motions using graphene strain sensors and actionlet ensemble model (Wang et al., 2014a, b). Varela et al. got excellent results from measuring the motion of knee and ankle of a human during walking using CaTraSys, a cable based parallel manipulator (Varela et al., 2015).

Above all, there have been some researches in falling down of the human, most of them focus on the aged people and the damage caused by falling. These researches are basically from the kinematic point of view which limits the application. Moreover, the studies about the impact in human fall are rarely reported. In this paper, an experimental layout consisting of motion capture system, accelerometer and force sensors is built, and a group of tests is carried out to acquire the information from human falling down. A fifteen markers model is established in the motion capture system to track the motion of the human, and two force sensor and two accelerometers are used to measure the status of the human when falling down. Thus corresponds to the two meaning of doing the experiments, namely, finding the kinematic and dynamic characteristics of motions from the necessary motion and impacting data. In fact, there are several ways to fall down for human, we examine two most common forms, falling forward and falling backwardin this paper. We use the forward and backward fall as the simplified model of human fall. The trajectories of primary points of human are evaluated, and motion strategies for reducing the impact force are summarized.

\section{Characteristics of human falling down}

Human falling down is common in daily life. Figure 1 shows the two most common types of a human falling down: falling backward and falling forward. In the Fig. 1a, the person fell backward, and in the Fig. 1b, the man fell forward. The fall of both of them is caused by lost balance, a possible reason for a fall in daily human life (Hsiao and Robinovitch, 1997). Lost balance leads to that human cannot recover to the standing posture, and the fall happens inevitably. Generally, a fall may consist of the following steps: Firstly, a person encounters a disturbance, such as a slip, an obstacle, or a sudden push. Secondly, the person tries to keep standing posture. If the attempt works, the person will not fall down. Otherwise, the person loses balance and falls down. Then, during the period of falling, the person uses his/her body motion, such as knee bending, waist bending and using the arm to contact the ground, to reduce the impact as far as possible. Finally, the person contacts on the ground and rolls on the ground to reduce the impact sometimes.

In this paper, we focus on the motion behavior and characteristics of a human falling in the situation when the falling cannot be avoided. In order to analyze the human falling after lost balance in detail, essential data of falling down should be acquired:

The detectable incipient motion.

- The acceleration changes during the falling.

- The trajectories of the main parts of a human.

- The motion after contacting on the ground.

- The motion strategies that can protect human from being hurt.

It is essential to recognize the detectable incipient motion, as it is the identification of whether a fall will happen or not. Through the acceleration changes, we can get the dynamic characteristics of the fall. The trunk and the head are two of the most critical components of the human body. It is reasonable to measure the variation of the acceleration of these 


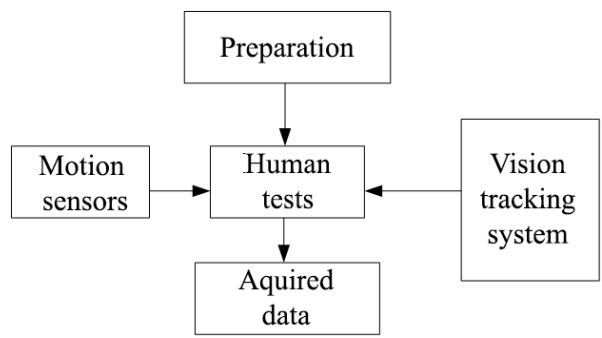

Figure 2. Structure of the experimental layout for testing procedure of human falling down.

two parts, especially at the time of impacting on the ground. The motion characteristic can be summarized by the trajectories of the main parts of the human body, such as the waist, back, knee, and ankle. Each motion, like bending the knee, can be expressed by the combination of different trajectories of body parts. The fall will not be over until the body stops to move. Thus, the motion after contacting on the ground is also essential for acquiring the characteristic of falling. We can summarize the motion and dynamic characteristics and propose some protection strategies, which can help us to understand the falling better.

\section{Experimental layout}

Experimental tests can be carried out to acquire the behaviors and characteristics of human motion in falling down. In this work an experimental layout with a measurement system has been settled up in the lab environment for monitoring purposes to understand configurations of human falling and to characterize numerical the motion. The measurement system consists of an optical motion capture system (from Motion Analysis Corp, Motion Analysis Corporation, 2010), two force sensors (from NITTA Corp.), and two accelerometers (from Xsens Corp.). Subjects were tested in this experimental environment by falling forward and backward. Motion trajectories were obtained by the optical motion capture system. Force sensors measured the impact force, and the acceleration of the head and chest was acquired by accelerometers. Detail experimental procedure will be introduced in the next section.

The structure of the experimental layout is designed as in the schemes in Fig. 2, four processes should be done for the tests for human, namely, preparation for the test, debugging the motion capture system and the accelerometers and the force sensors, and arranging the lab environments.

The sequence of procedure for the proposed tests is illustrated in the Fig. 3. The test includes three main sections: preparation of the test, conducting a test and the numerical analysis of the test results. The preparation consists of calibrating the sensors and the motion capture system and guiding the subjects to run the test in a standard way. Then, the tests are conducted under the direction of the operators for

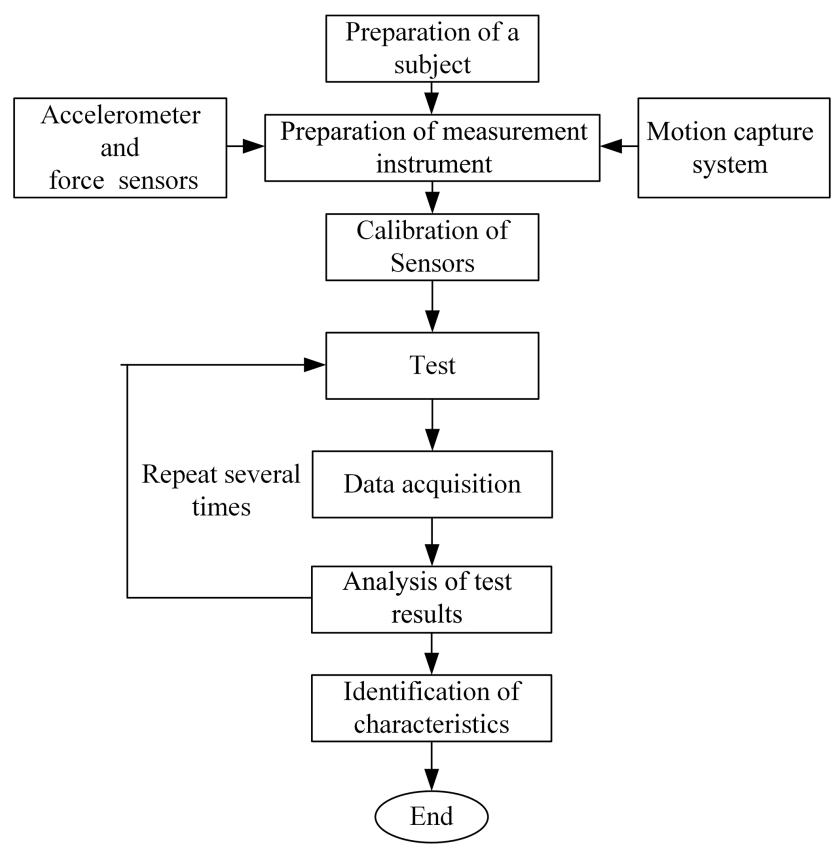

Figure 3. Flowchart for an experimental testing procedure of human falling down.

several times. During each test, the motion data are obtained from the motion tracking system. The last section is to analyze the acquired data for an evaluation of the test of falling down.

The experimental layout is explained in Fig. 4. As is shown in the Fig. 4, a gymnastic mattress with a thick of $30 \mathrm{~cm}$ was located on the ground as a landing surface for the fall. During all trials, the subjects stood on a stage at the front of the gymnasium mattress and then fell backward and forward. A 12-camera motion capture system was used to trace the motion trajectory of the objects from three-dimension. The frequency of Motion Capture System is $60 \mathrm{~Hz}$. The resolution of this system is $0.01 \mathrm{~mm}$. A regular video camera from side view was adopted to record the whole process of the falling. When human fall forward or backward, the entire chest or back impact on the mattress at different time. In order to detect the force during the whole process of the human fall, at least two force sensor should be used. During each trial, two force sensors were put on the mattress to measure the contact force between the subjects and mattress. As the frequency of the Motion Capture System is not fast enough to compute the acceleration the moment of contact, two accelerometers attached to the subjects' chest and head, which are the two of the most important parts of the human body, were used to measure the acceleration during the falling. The frequency of the acceleration sensor is $100 \mathrm{~Hz}$. All the sensors were connected to one PC by CAN bus so that the acquired data can be synchronous. 


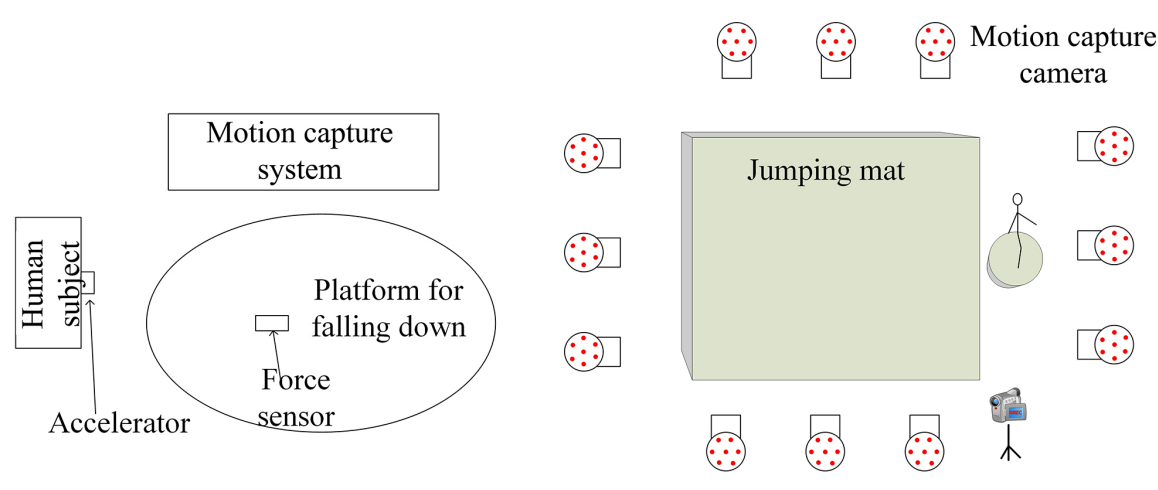

(a)

(b)

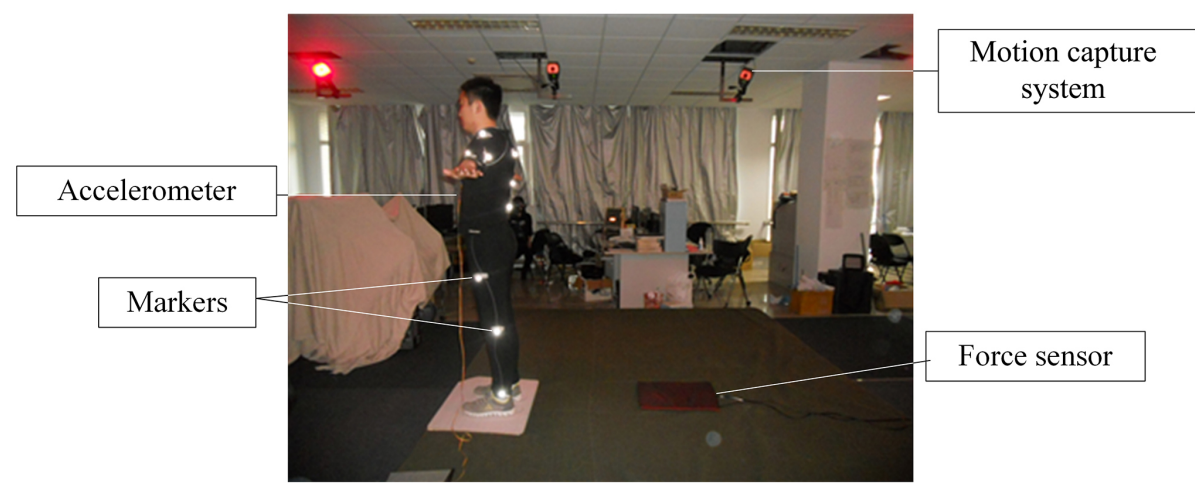

(c)

Figure 4. Experimental layout at BIT lab in Beijing: (a) a general top view scheme; (b) a scheme of lab arrangement; (c) a photo of the lab arrangement.

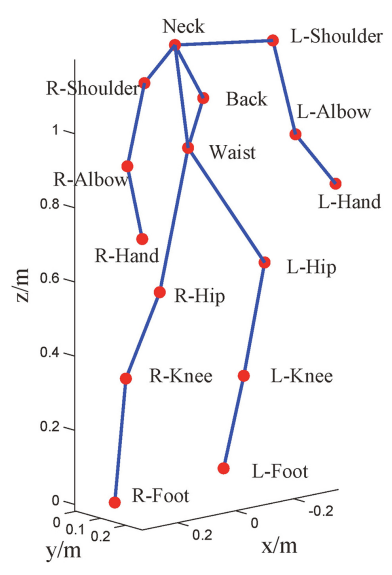

(a)

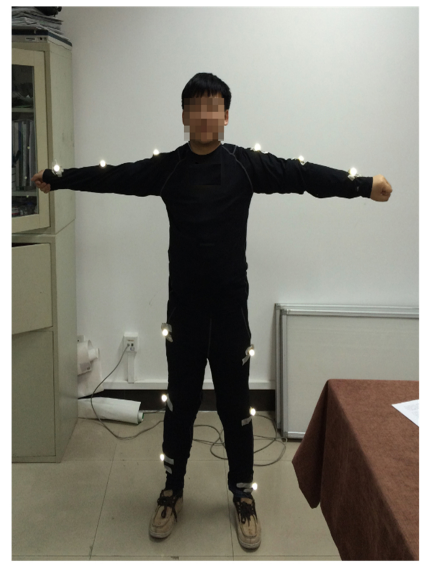

(b)

Figure 5. Mode of testing human backward falling down: (a) a scheme with sensors location; (b) a lab test.

\section{Testing modes}

In this paper, results are reported for the case of human falling down forward and backward. In order to obtain the data of human falling down, five healthy subjects (age 24-26 years old, height $168-173 \mathrm{~cm}$, weight $65-70 \mathrm{~kg}$, male and female) were recruited in the test activity for the research from Beijing Institute of Technology (see Table 1). 15 spherical markers were attached to the subject's neck, shoulders, elbows, hands, back, waist, hips, knees, and ankle. In order to track the markers without interference, a special cloth with a dark color is recommended (see Fig. 5b). This suit, including a tight coat and trousers, keeps the markers still relative to the subject. A human model based on 15 segments was built as Fig. 5 in the Motion Capture System. Basically, 15 markers can be considered enough to describe the falling motion of this work. The reason why we use the 15 markers model is that the 15 markers model has several advantages:

- Simple computation.

- Compact representation.

- Main segments of human body (leg, trunk and arm). 

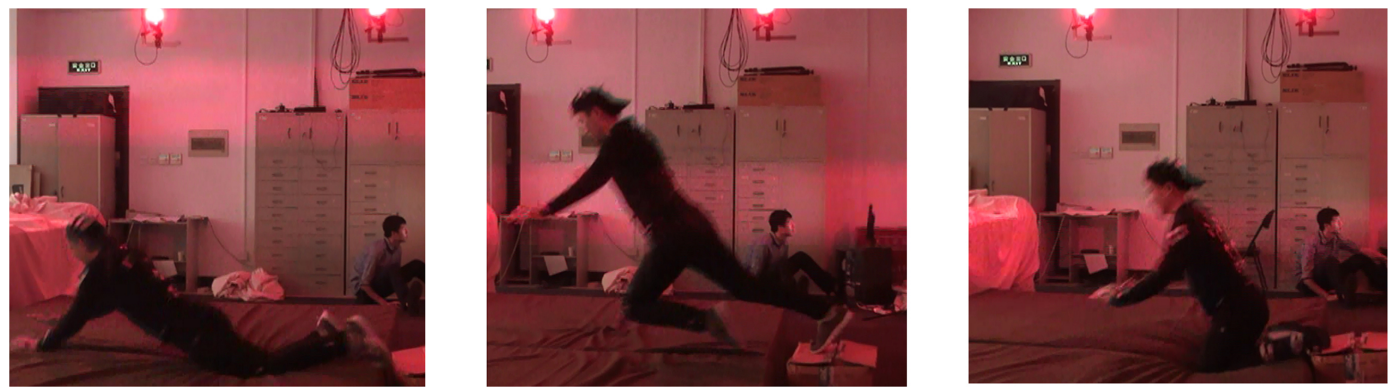

Figure 6. Different ways of falling forward.
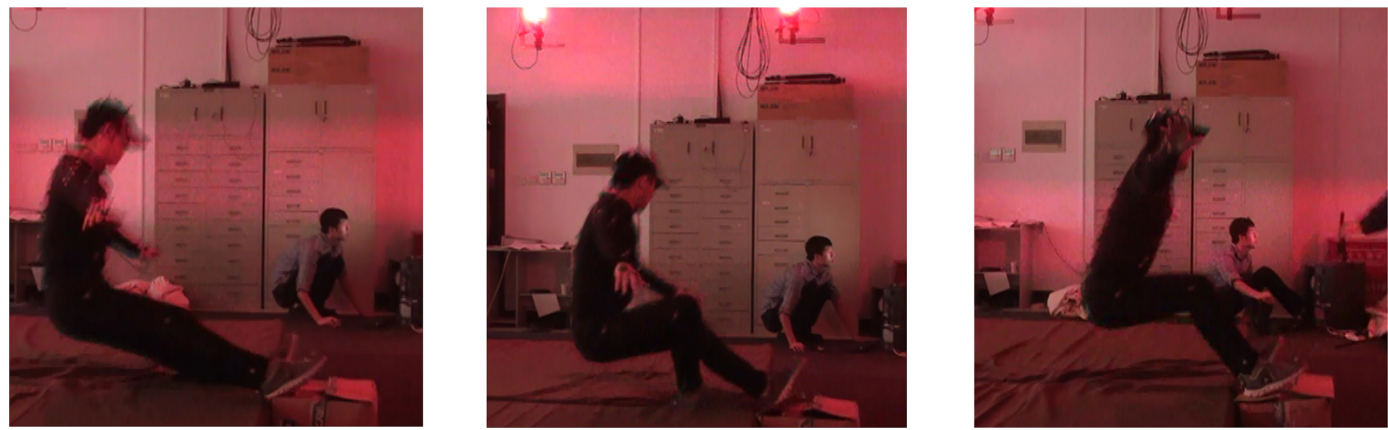

Figure 7. Different ways of falling backward.

Table 1. The parameters of the subjects.

\begin{tabular}{lrrr}
\hline Subjects Number & Age & Weight $(\mathrm{kg})$ & Height $(\mathrm{m})$ \\
\hline 5 & $24-26$ & $65-70$ & $1.68-1.73$ \\
\hline
\end{tabular}

- Main DOFs of human body.

In Fig. 8, a model for falling test is described. The falling test procedure is shown in the Fig. 9. Before the test, the testers interpreted the detailed procedure to the subjects as follows: All the falls were initiated from a standing position (see Fig. 9a). When a test began, the subject should be asked "are you ready?" The subject would fall down by a sudden push within a random time (1-15 s) after he or her gave the answer "Yes" (see Fig. 9b). Figures 8c and 9d illustrate the following configuration of falling down: The subject controls body motion to make a relatively safe way to contact on the mattress. After impacting on the mattress, the subject reaches the final configuration (see Fig. 9d). Since the fall is heavily affected by the pushing force, three factors are taken into consideration: (1) All the subjects shouldn't be hurt by the tests. (2) All the experiments should be end with the human falling. (3) The pushing force in different tests should be basically the same. The testers and subjects had practiced to how to push and fall for several times before the tests. Figures 6 and 7 show different ways to fall forward and backward. The third falling motion in the Figs. 6 and 7

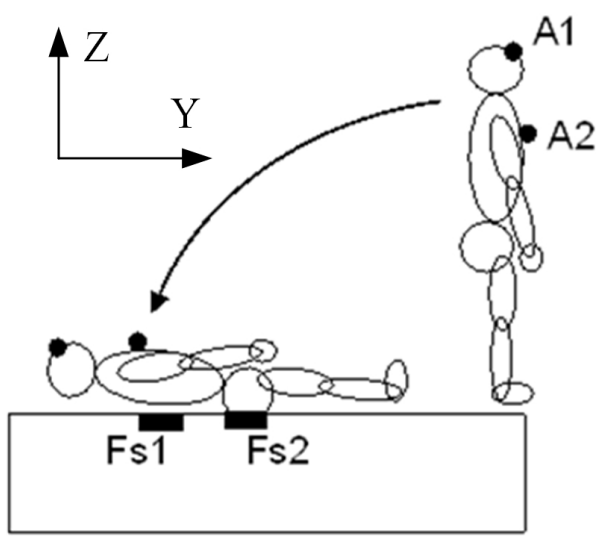

Figure 8. A model for the lab test of human falling down.

were selected as the fall motion in the final tests because that they had the smallest impact force compared the other falling styles.

Each repeated eight times for falling backward and forward, respectively.

\section{Results of tests}

In this section, the results of the test are reported and analyzed as based on the data acquired from the motion capture system, force sensors, and accelerometers. The markers of back, waist, hip, and ankle are selected to interpret the char- 


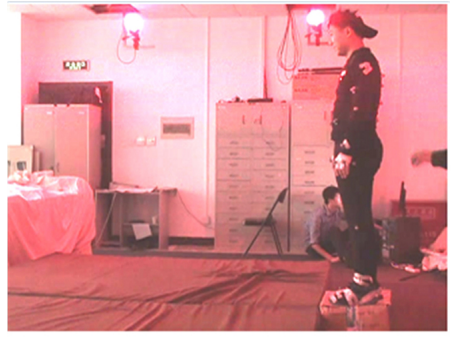

(a)

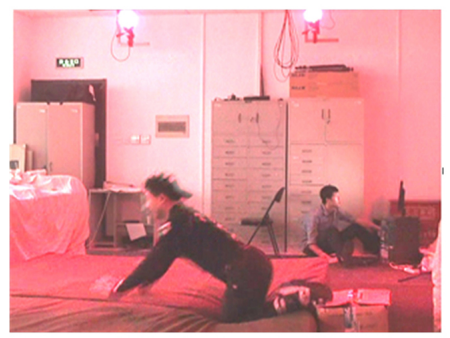

(d)

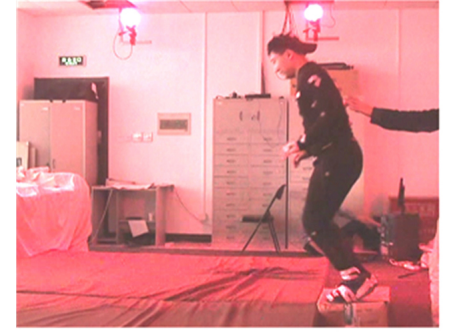

(b)

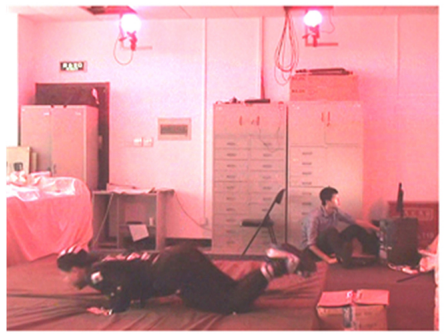

(e)

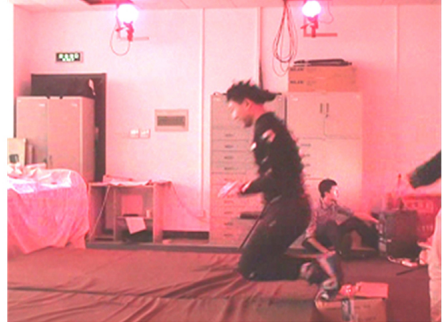

(c)

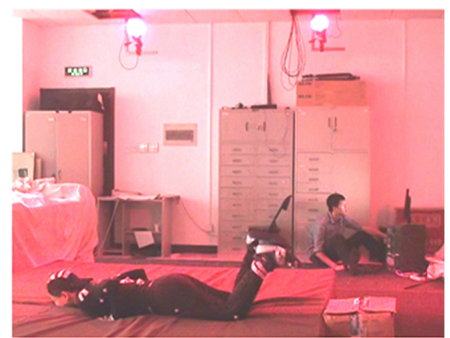

(f)

Figure 9. A snapshot of a lab test of a forward human falling down: (a) initial standing up; (b) incipient falling; (c) configuration during the falling; (d) impact on the mattress; (e) absorbing the impact; (f) final configuration.

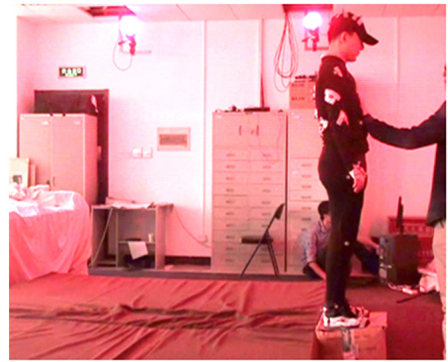

(a)

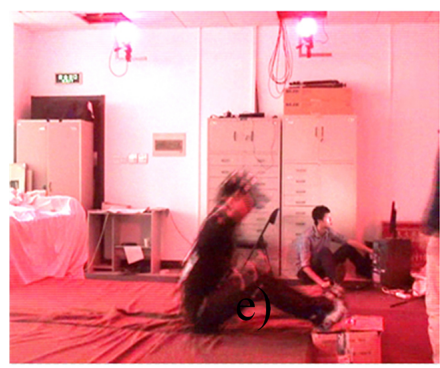

(d)

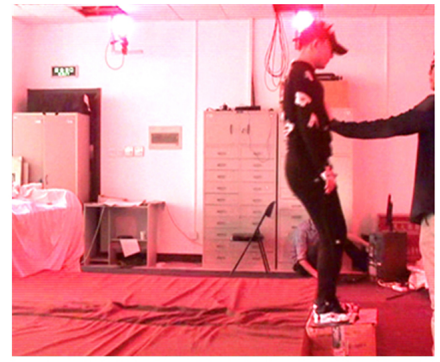

(b)

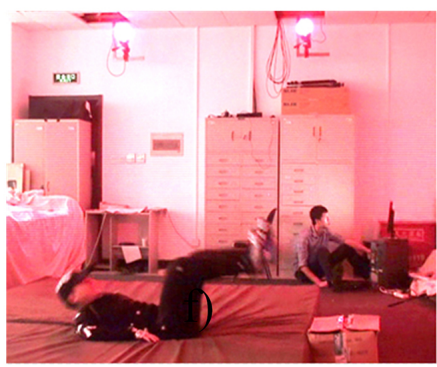

(e)

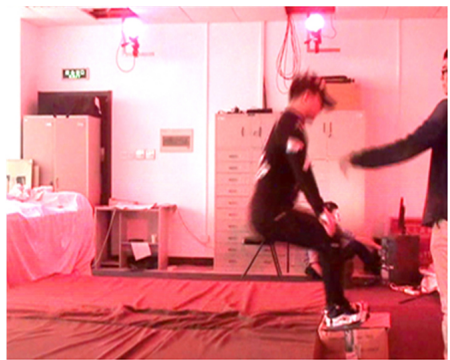

(c)

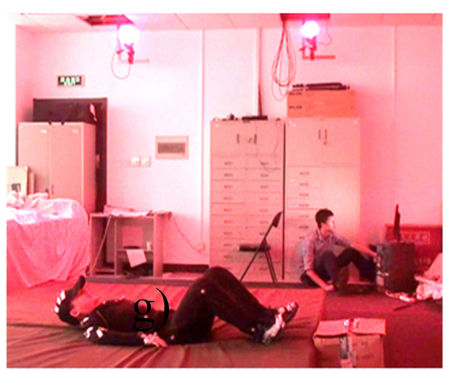

(f)

Figure 10. A snapshot of a lab test of backward human falling down: (a) initial standing up; (b) incipient falling; (c) configuration during the falling; (d) impact on the mattress; (e) absorbing the impact; (f) final configuration.

acteristic of a human falling in the sagittal plane. The body motion is to be discussed in detail in this section using numerical analysis. The results discussed in the following are from the mean of all the acquired data. We use the time the impact of the subject on the mattress as the reference time to synchronize the motion data from different subjects.

Figures 9 and 10 are snapshots of the falling backward and forward of one test. Figures 9c, d and 10c, d show the subject changed his body gait to adjust the landing parts to the 


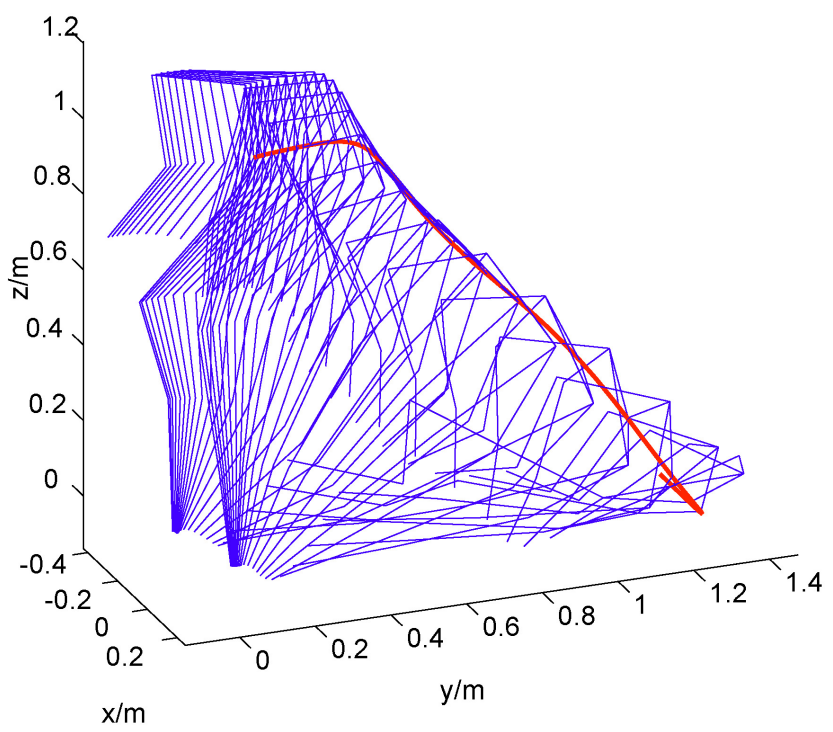

Figure 11. Evolution of the human model during the backward falling down of the test in Fig. 8 by using the body segments (red line is the trajectory of back marker).

mattress. The hip was the most frequent landing parts among the tests of falling backward, while the knee was landing part among the tests of falling forward. This motion corresponds that the hip is one of the softest and thickness parts of the body, and has tough impact resistant. Figures $9 \mathrm{e}, \mathrm{f}$ and $10 \mathrm{e}$, f show the motion after the subject touched on the mattress. In the backward falling, the subject rolled on the mattress after landing. This motion could extend the impact time as well as reduce the impact force. And in the forward falling, the subject used the hands to absorb the impact force. These motions will be explained from the numerical analysis in the following part.

Figures 11 and 12 show the evolution of the human model during the backward and forward falling down using the body segment, respectively. Each segment was drawn by the trajectories of two markers on the subject's body. The subject fell down with the postural adjustment instead of falling directly. At the beginning of both of the backward and forward falling, the height of the trunk was nearly unchanged. This corresponds to the incipient phase (Figs. 9b and 10b) of the fall and indicates that the subject tried to keep body balance from falling down. As the fall continued, the slope of the trajectory of the back increased. This was due to the motion of knee bending and waist bending, which shortened the distance between the ankle and the trunk (Figs. 9c, d and $10 \mathrm{c}, \mathrm{d})$. At the end of the fall, the trajectory of the back had a sudden bounce. This corresponds the elasticity of the landing surface, and may not happen during a falling on the ground.

The acquired values of the accelerometer attached to the subject's chest and head are shown in the Figs. 11 and 13. The red and blue curve represent the acceleration the chest and head, respectively. Both of them have similar characteris-

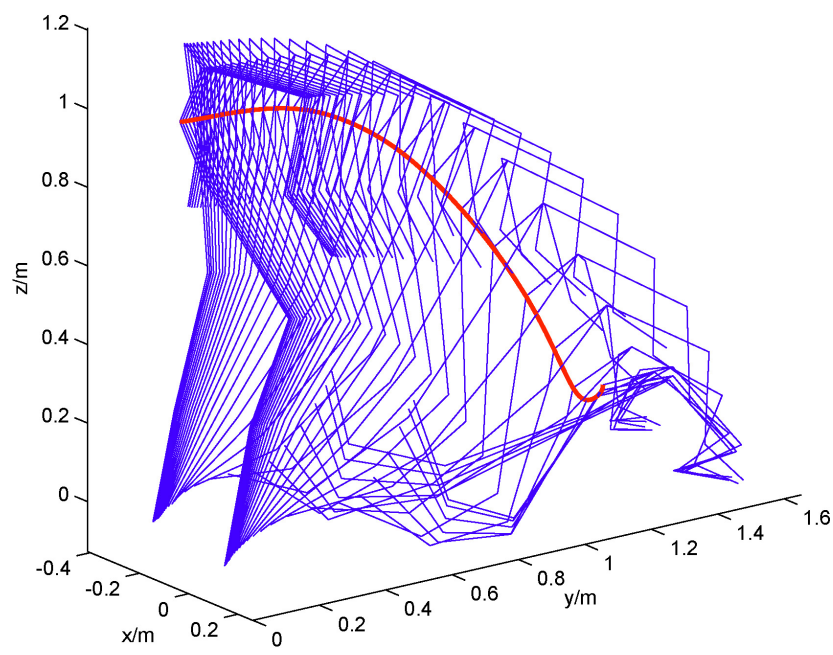

Figure 12. Evolution of the human model during the forward falling down of the test in Fig. 7 by using the body segments (red line is the trajectory of back marker).

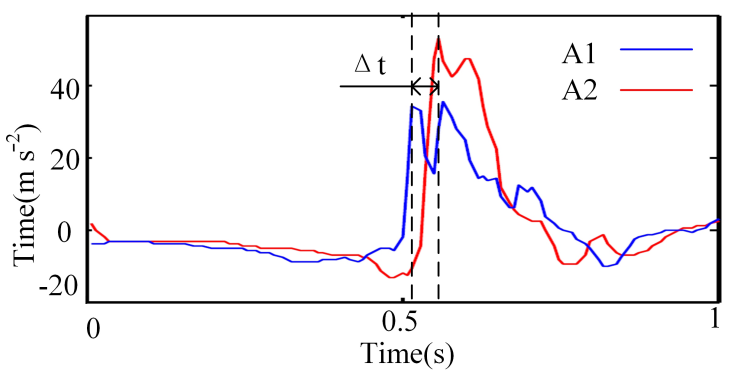

Figure 13. Acquired measurements of the backward falling down acceleration in the sensor A of the layout in Fig. 1 during the test in Fig. 8.

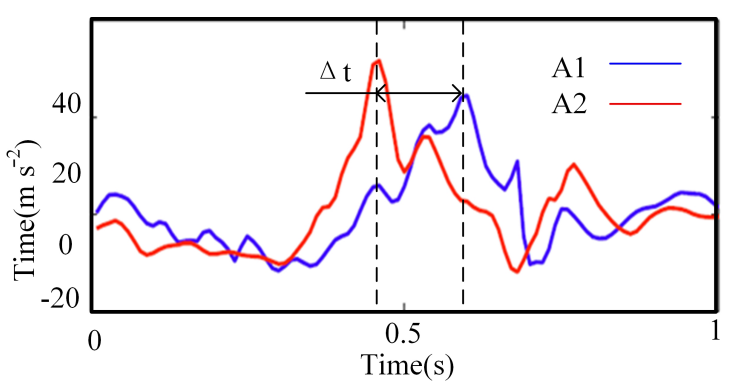

Figure 14. Acquired measurements of the forward falling down acceleration in the sensor A of the layout in Fig. 1 during the test in Fig. 7.

tics. The acceleration increased after the beginning of falling down and had a massive changing when the subject impacted on the mattress. In both of the two kinds of falling, the time between the beginning of falling and impact on the mattress is about $0.5 \mathrm{~s}$. Even though the landing surface is a soft mattress, the max acceleration reaches almost $6 \mathrm{~g}$. It is to note 


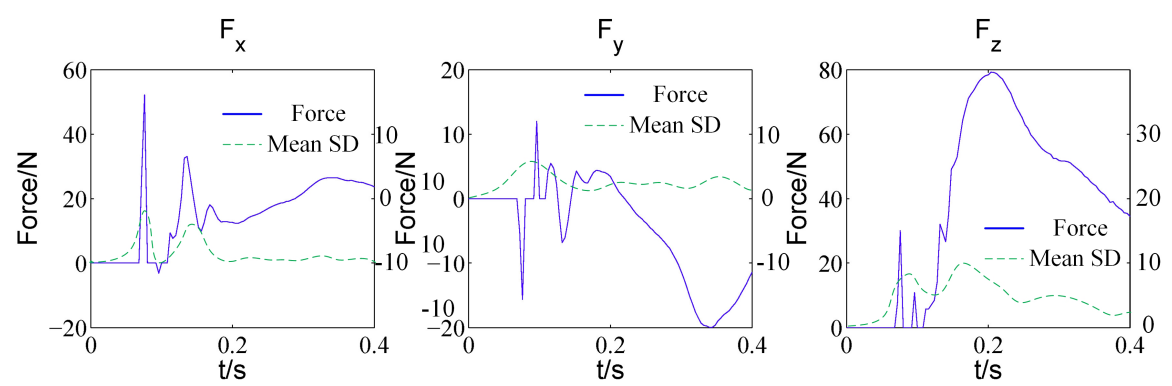

(a)
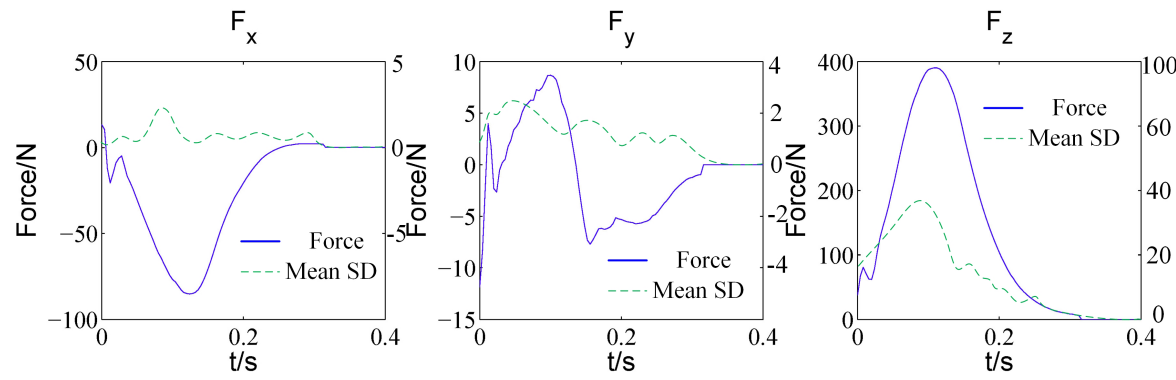

(b)

Figure 15. Acquired measurements of the backward falling down in the sensor FS1 and FS2 of the layout in Fig. 1 during the test in Fig. 8: (a) components of the force of FS1; (b) components of the force of FS2.

that the sudden acceleration change is measured over a period of about $0.2 \mathrm{~s}(\Delta t)$. This 0.2 second is nearly the same as the time of the impact between the subject and the landing mattress. Particularly, the movement range of the head was smaller than the chest. It is due that the head is the most important parts of the body, and during all the tests, the head was observed hardly to impact on the mattress.

The Figs. 15 and 16 show the force that was acquired from the two force sensors above the mattress. In order to show the repeatability of the tests, mean standard deviation was calculated (red line in each picture). The consistency is within acceptable ranges. We only focus on the moment around the impact ( $0.1 \mathrm{~s}$ before the impact, $0.3 \mathrm{~s}$ after the impact). In the backward falling, the subject impacted the force sensor Fs1 about $0.1 \mathrm{~s}$ after the force sensor Fs2, and the contact time impacting each sensor lasts about 0.2 to $0.3 \mathrm{~s}$. The short period shows the rolling motion on the mattress, a protection strategy, to resist the impact injuries (Fig. 8d and e).

The force in $x$ and $y$ direction vibrated near the zero after impact. This indicates that slight rotation happened after the subject impacted on the mattress. In the forward falling, the force was much smaller than the force measured from the backward falling, and the magnitude of Fs1 and Fs2 were nearly the same. This is due to that the subject contacted the landing surface with the knee and then the hand, most of the impact force was absorbed. It is to note that compared to the force in the $z$ direction, the impact force in the $x$ and $y$ direction are very small, this indicates that the protection should pay more attention on the collision rather than the rotation.
Nevertheless, the lateral force cannot be neglected. The time evaluation of the acquired force the impact shows that the impact is not instantaneous and it lasts with accommodation of the body with the distribution of the actions, although it is evident the first impulsive contact.

In order to emphasize the difference of the motion of main parts of the body, the trajectories of ankle, hip, knee and back were compared in the sagittal plane in Figs. 17 and 18 in backward and forward, respectively. The trajectories are in the sagittal plane. The arrows represent the motion direction. The red, blue, and green curves show the trajectories of the ankle, hip, and knee, respectively. The trajectory of the back is not a complete circle that rotates around the ankle, which indicates that the subjects used the body motion to change his/her gaits. In all the tests, the motion of the hip and the back had the nearly same trajectories. The relative significant difference exists between the trajectories knee and other makers, due to the knee bending and waist bending. In both of the forward and backward falling, the ankle lifted into the air from the ground while the body was falling down (Figs. 9e and 10e). It helped the subject to finish the rolling motion after contacted on the landing surface and reduce the impact force in the backward falling, due to that the rolling motion increases the impact time.

This motion made the waist impact before the back, thus had been improved by the time interval of two force sensors, and resisted severe damage of head.

Figures 19 and 20 show the computed angle of the knee and hip joint, respectively. The angles are computed by the 

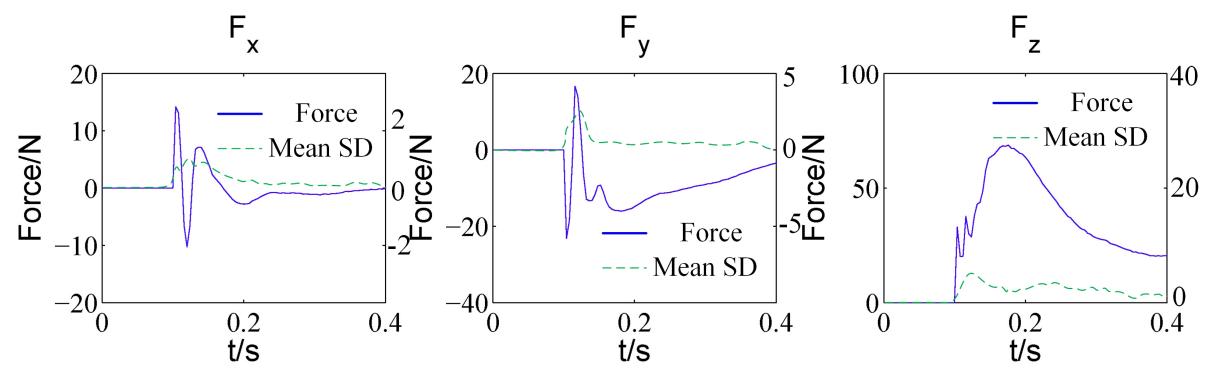

(a)
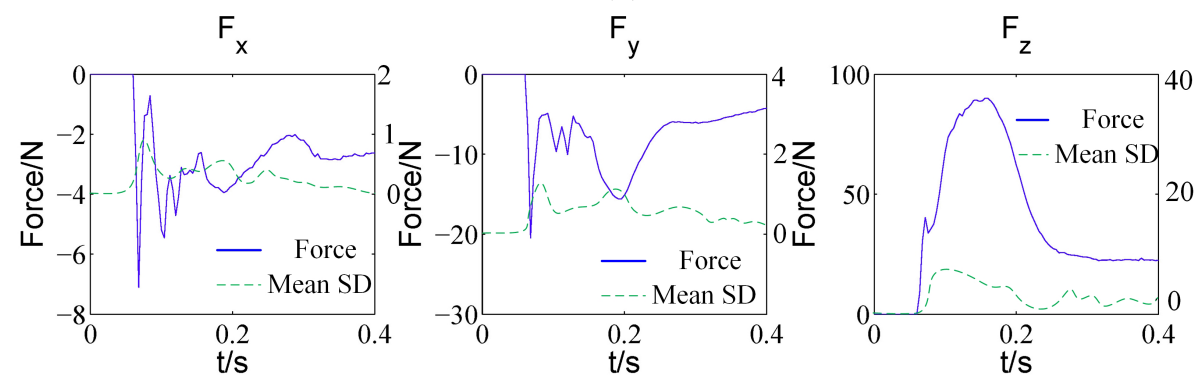

(b)

Figure 16. Acquired measurements of the forward falling down in the sensor FS1 and FS2 of the layout in Fig. 1 during the test in Fig. 8: (a) components of the force of FS1; (b) components of the force of FS2.

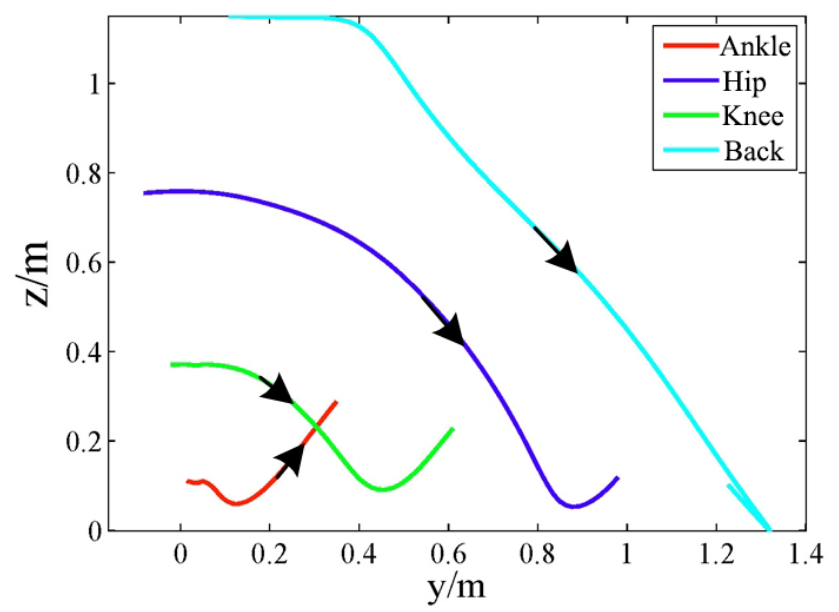

Figure 17. Trajectories of reference points of the ankle, back, hip and knee in the sagittal plane in the test of falling backward in Fig. 8.

Eqs. (1) and (2):

$\theta_{\text {knee }}=\cos ^{-1}$

$\left(\begin{array}{c}\left(Y_{\text {hip }}-Y_{\text {knee }}\right)^{2}+\left(Z_{\text {hip }}-Z_{\text {knee }}\right)^{2}+\left(Y_{\text {ankle }}-Y_{\text {knee }}\right)^{2} \\ \frac{+\left(Z_{\text {ankle }}-Z_{\text {knee }}\right)^{2}-\left(Y_{\text {hip }}-Y_{\text {ankle }}\right)^{2}-\left(Z_{\text {hip }}-Z_{\text {ankle }}\right)^{2}}{2 \sqrt{\left(Y_{\text {hip }}-Y_{\text {knee }}\right)^{2}+\left(Z_{\text {hip }}-Z_{\text {knee }}\right)^{2}} \cdot \sqrt{\left(Y_{\text {ankle }}-Y_{\text {knee }}\right)^{2}+\left(Z_{\text {ankle }}-Z_{\text {knee }}\right)^{2}}}\end{array}\right)$

$\theta_{\text {hip }}=\cos ^{-1}$

$\left(\begin{array}{c}\left(Y_{\text {hip }}-Y_{\text {knee }}\right)^{2}+\left(Z_{\text {hip }}-Z_{\text {knee }}\right)^{2}+\left(Y_{\text {hip }}-Y_{\text {back }}\right)^{2} \\ \frac{+\left(Z_{\text {hip }}-Z_{\text {back }}\right)^{2}-\left(Y_{\text {back }}-Y_{\text {knee }}\right)^{2}-\left(Z_{\text {back }}-Z_{\text {knee }}\right)^{2}}{2 \sqrt{\left(Y_{\text {hip }}-Y_{\text {knee }}\right)^{2}+\left(Z_{\text {hip }}-Z_{\text {knee }}\right)^{2}} \cdot \sqrt{\left(Y_{\text {hip }}-Y_{\text {back }}\right)^{2}+\left(Z_{\text {hip }}-Z_{\text {back }}\right)^{2}}}\end{array}\right)$

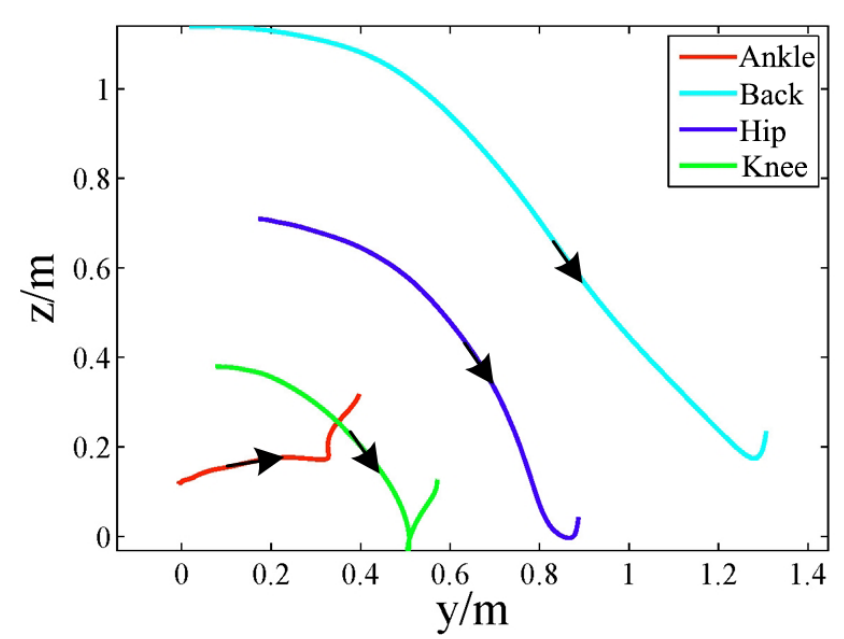

Figure 18. Trajectories of reference points of ankle, back, hip and knee in the sagittal plane in the test of falling forward in Fig. 7.

where, the $\left(X_{\text {hip }}, Y_{\text {hip }}, Z_{\text {hip }}\right),\left(X_{\text {knee }}, Y_{\text {knee }}, Z_{\text {knee }}\right),\left(X_{\text {back }}\right.$, $\left.Y_{\text {back }}, Z_{\text {back }}\right)$ stand for the coordinates of the hip knee and back, respectively.

In the backward falling, the angles variation range of the hip joint is larger than that of the knee joint. The joints reached the maximum angle at a different time. It can be concluded that the knee bending motion began firstly, and then stretched. These motions also could be seen from the Fig. $10 b$ to d. 
Table 2. Summary of acquired date for characteristics of human falling down.

\begin{tabular}{lrrrrrr}
\hline Parameters & $A_{\mathrm{ccH}}\left(\mathrm{m} \mathrm{s}^{-2}\right)$ & $\mathrm{Acc}_{\mathrm{T}}\left(\mathrm{m} \mathrm{s}^{-2}\right)$ & $F_{1 z}(\mathrm{~N})$ & $F_{2 z}(\mathrm{~N})$ & $\left.\theta_{\text {knee }}{ }^{(}\right)$ & $\left.\theta_{\text {waist }}{ }^{\circ}\right)$ \\
\hline Backward falling & $-6-38$ & $-10-52$ & $0-80$ & $0-400$ & $120-180$ & $80-180$ \\
\hline
\end{tabular}

(a)

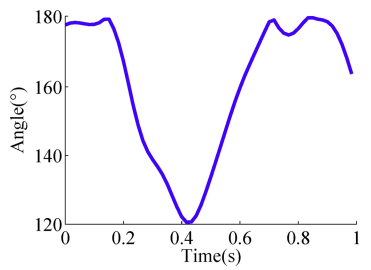

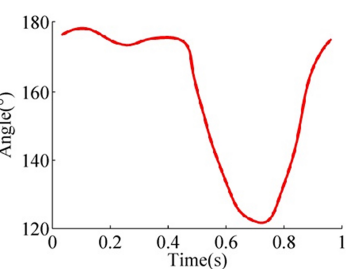

(b)
Figure 19. The computed angle of the knee joint and waist joint when falling backward in the test of Fig. 8: (a) the angle of knee, (b) the angle of waist.

The case in forward falling was opposite. The angle variation of the knee joint was larger than the hip joint. The maximum angle of the knee reached about $60^{\circ}$, and the waist was about $80^{\circ}$. These two joints reached the maximum angle at nearly the same moment when the subject landed on the mattress (Fig. 9d). Then, the subject stretched the waist and knee to contact the mattress with the trunk. It resulted the force measured by the force sensors were not as large as the force in the backward falling.

The average value of the main parameters, which represent the characteristics of the human falling down, is summarized in Table 2.

The range of the acceleration of the forward and backward falling is almost the same. It is noticed that acceleration of the head $\left(\mathrm{Acc}_{\mathrm{H}}\right)$ is smaller than that of the trunk $\left(\mathrm{Acc}_{\mathrm{T}}\right)$ in both situations. In order to ensure the head safety, the subjects tried to protect the head from the first impact on the landing surface. This helped to reduce the sudden change of the acceleration of the head.

The main difference between the forward and the backward falling is in the knee and waist motion. In the forward falling, the knee had a larger range $\left(\theta_{\text {knee }}\right)$ of motion than the waist $\left(\theta_{\text {waist }}\right)$. In the backward falling, it is just the opposite. The data acquired from the force sensors $\left(F_{1 z}\right.$ and $\left.F_{2 z}\right)$ also confirm this difference. The impact force of the trunk was much smaller than that of the waist in the backward falling due to that the waist impacted on the landing surface first. While in the forward falling, both of the impact force of trunk and waist were small as a result of that the knee and arm contacted the mattress first, and absorbed most of the impact force.

Nevertheless, the combination motion of the knee and the waist generated the same results: impacting on the landing surface with the strongest and muscular parts of the body. Thus, the impact force could be resisted and absorbed, which

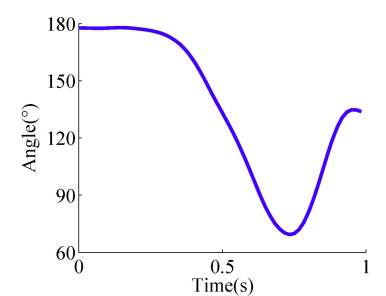

(a)

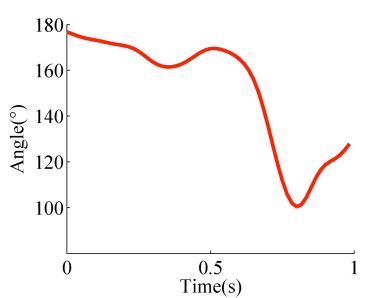

(b)
Figure 20. The computed angle of the hip joint when falling forward in the test of Fig. 7: (a) the angle of knee, (b) the angle of waist.

is the most distinct characteristic of the human falling down. We can summarize some protection strategies based on the motion and dynamic characteristics mention above as following:

- Forward falling: Using the knees as the first place to touchdown can reduce the falling momentum. The impact force of the chest can be reduced to a small value (less than $100 \mathrm{~N}$ ) by using the hands.

- Backward falling: The hip can absorb most of the impact force (about $400 \mathrm{~N}$ ). A rolling movement after the impact is recommended to reduce the vertical impact force. Body balance after impact can be kept by the arm motion.

- Forward and backward falling: Bending the knee and hip joint to lower the center of mass is an effective movement to reduce the impact force and acceleration changing. The arm is a secondary choice to absorb the impact force because it has relative fragile structure.

\section{Conclusions}

In this paper, a motion data acquired system was used for the tests of human falling. This system consists of motion capture system, accelerometer sensors, and force sensors. Two kinds of falling, backward and forward, were taken into consideration. The experimental tests and data acquisition were conducted with a detailed procedure. Results from the tests were elaborated to interpret the characteristics of the human falling down. Angles of knee and hip joints were computed for quantitative analysis the motion of human falling. Some protection strategies based on the motion and dynamic characteristics mention are proposed in order to better understand 
the human falling. The paper shows the characteristic motions and actions in human falling down with a numerical characterization of human parameters.

Data availability. All the data in this manuscript can be obtained by contacting the corresponding author.

\section{The Supplement related to this article is available online at doi:10.5194/ms-8-79-2017-supplement.}

Competing interests. The authors declare that they have no conflict of interest.

Acknowledgements. This work was supported in part by the National Natural Science Foundation of China under Grant 61533004, 61320106012, 61375103, and 61321002, in part by the 863 Program of China under Grant 2015AA043202, and 2015AA042305, in part by the Key Tech. R\&D Program under Grant 2015BAF13B01 and 2015BAK35B01, the Beijing Municipal Science and Technology Project under Grant D161100003016002, and in part by the "111" Project under Grant B08043.

Edited by: A. Müller

Reviewed by: J. Solis and three anonymous referees

\section{References}

Ayusawa, K., Ikegami, Y., and Nakamura, Y.: Simultaneous global inverse kinematics and geometric parameter identification of human skeletal model from motion capture data, Mech. Mach. Theory, 74, 274-284, 2014.

Chen, K., Trkov, M., Yi, J., Zhang, Y., Liu, T., and Song, D.: A robotic bipedal model for human walking with slips. In Robotics and Automation (ICRA), 2015 IEEE International Conference on (pp. 6301-6306). IEEE, 2015.

Gross, R. and Shi, J.: The cmu motion of body (mobo) database, 2001.

Gupta, J. P., Dixit, P., Singh, N., and Semwal, V. B.: Analysis of Gait Pattern to Recognize the Human Activities, arXiv preprint arXiv:1407.4867, 2014.

Hitcho, E. B., Krauss, M. J., Birge, S., Claiborne Dunagan, W., Fischer, I., Johnson, S., and Fraser, V. J.: Characteristics and circumstances of falls in a hospital setting, J. Gen. Intern. Med., 19, 732-739, 2004.

Hsiao, E. T. and Robinovitch, S. N.: Common protective movements govern unexpected falls from standing height, J. Biomech., 31, 1-9, 1997.

Huang, Q., Yu, Z., Zhang, W., Xu, W., and Chen, X.: Design and similarity evaluation on humanoid motion based on human motion capture, Robotica, 28, 737-745, 2010.
Lee, Y. S. and Chung, W. Y.: Visual sensor based abnormal event detection with moving shadow removal in home healthcare applications, Sensors, 12, 573-584, 2012.

Ma, G., Huang, Q., Yu, Z., Chen, X., Hashimoto, K., Takanishi, A., and Liu, Y. H.: Bio-inspired falling motion control for a biped humanoid robot. In Humanoid Robots (Humanoids), 2014 14th IEEE-RAS International Conference on (pp. 850-855), IEEE, 2014.

Robinovitch, S. N., Brumer, R., and Maurer, J.: Effect of the "squat protective response" on impact velocity during backward falls, J. Biomech., 37, 1329-1337, 2004.

Roos, P. E. and Dingwell, J. B.: Using dynamic walking models to identify factors that contribute to increased risk of falling in older adults, Hum. Movement Sci., 32, 984-996, 2013.

Sandler, R. and Robinovitch, S.: An analysis of the effect of lower extremity strength on impact severity during a backward fall, J. Biomech. Eng., 123, 590-598, 2001.

Schmitz, A., Ye, M., Shapiro, R., Yang, R., and Noehren, B.: Accuracy and repeatability of joint angles measured using a single camera markerless motion capture system, J. Biomech., 47, 587591, 2014.

Tan, J. S., Eng, J. J., Robinovitch, S. N., and Warnick, B.: Wrist impact velocities are smaller in forward falls than backward falls from standing, J. Biomech., 39, 1804-1811, 2006.

Tomii, S. and Ohtsuki, T.: Falling detection using multiple doppler sensors, in: e-Health Networking, Applications and Services (Healthcom), 2012 IEEE 14th International Conference on (pp. 196-201), IEEE, 2012.

Users' manual, Motion Analysis Corporation, Santa Rosa, CA, USA, 2010.

Varela, M. J., Ceccarelli, M., and Flores, P.: A kinematic characterization of human walking by using CaTraSys, Mech. Mach. Theory, 86, 125-139, 2015.

Wang, J., Liu, Z., Wu, Y., and Yuan, J.: Learning actionlet ensemble for 3D human action recognition. Pattern Analysis and Machine Intelligence, IEEE Transactions on, 36, 914-927, 2014a.

Wang, Y., Wang, L., Yang, T., Li, X., Zang, X., Zhu, M., and Zhu, H.: Wearable and highly sensitive graphene strain sensors for human motion monitoring, Adv. Funct. Mater., 24, 4666-4670, 2014b.

Yang, S. X., Christiansen, M. S., Larsen, P. K., Alkjær, T., Moeslund, T. B., Simonsen, E. B., and Lynnerup, N.: Markerless motion capture systems for tracking of persons in forensic biomechanics: an overview, Computer Methods in Biomechanics and Biomedical Engineering: Imaging \& Visualization, 2, 46-65, 2014.

Yang, Y., Schonnop, R., Feldman, F., and Robinovitch, S. N.: Development and validation of a questionnaire for analyzing real-life falls in long-term care captured on video, BMC geriatrics, 13, p. 40, 2013.

Zhao, J., Xie, B., and Song, C.: Generating human-like movements for robotic arms, Mech. Mach. Theory, 81, 107-128, 2014.

Zielinska, T., Chew, C. M., Kryczka, P., and Jargilo, T.: Robot gait synthesis using the scheme of human motions skills development, Mech. Mach. Theory, 44, 541-558, 2009. 